\title{
A Study on the [AB+! ]- a Form of V+O Combining Separable Slot
}

\author{
Chunling $\mathrm{Li}^{1 \text {, a }}$, Xiaoxiao Wang ${ }^{2, b}$ \\ ${ }^{1}$ College of Liberal Arts, Shenyang Normal University, China \\ ${ }^{2}$ College of International Education, Shenyang Normal University, China \\ achl4019@sina.com, b yuanxiao8211@hotmail.com
}

Keywords: $\mathrm{V}+\mathrm{O}$ form;separable word;separable slot;semantic feature

\begin{abstract}
This paper combines semantics and syntax to study the combining separable slots of $\mathrm{V}+\mathrm{O}$ separable words in modern Chinese in a comprehensive perspective, namely the $[\mathrm{AB}+!$ ] form. The paper discusses and analyzes the selection and restriction within the quantitative $\mathrm{V}+\mathrm{O}$ separable words. It further analyzes the internal semantic features and rules of use for separable words falling into this category, the outcome of the study can serve as a reference for computer linguistics and Chinese teaching.
\end{abstract}

\section{Introduction}

$\mathrm{V}+\mathrm{O}$ separable words $\mathrm{AB}$ are unique grammatical phenomena in modern Chinese language. The features of separable words lie in the fact that their meanings are generated in the same way as other composite words, but their forms are similar to phrases. Thus it is in the middle between word and phrase. It is not only difficult for ontological study of language, but also key to Chinese teaching and computer linguistics.

"Slot" was first used as a grammatical term by Yuanren Zhao(1979). Zhao pointed out that grammar is the study of groups or classes of forms as regards their occurrence in frames or slots constituted by other classes. All forms which behave alike in this respect are members of a form class. ${ }^{[1]}$ Different grammatical phenomena of separable words are thereby controlled by a special "slot". This paper concludes all sorts of separable forms of disyllable $\mathrm{V}+\mathrm{O}$ separable words $\mathrm{AB}$ as "separable slots". ${ }^{[2]}$ Separable slots are consisted of discrete slots and combining slots. They together construct all grammatical forms of separable words in Chinese, namely the system of separable slots. The relation of this hierarchical system is: separable slot $\rightarrow$ separable pattern slot $\rightarrow$ separable paradigm slot. Two of these three sub systems are abstract and the other is concrete as shown below.

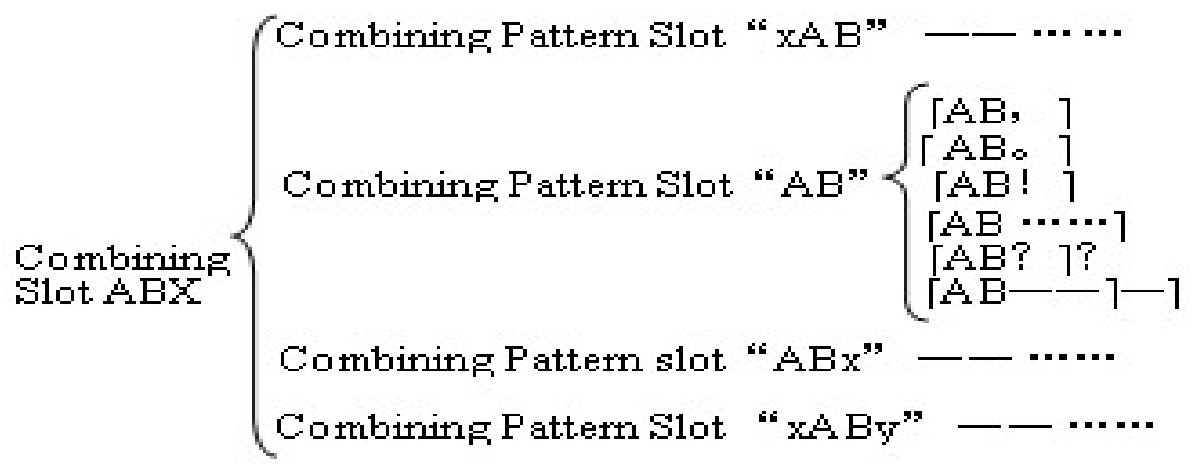

${ }^{[1]}$ Yuanren Zhao. A Grammar of Spoken Chinese [M]. Commercial Press, Beijing. 1979, pp.7.

${ }^{[2]}$ Here the "slot" is not completely equal to the "slot" defined by Yuanren Zhao, and is also not equal to "frame" and "form" in grammar study field. The "slot" here refers to a hierarchy which includes separable slot, pattern slot and paradigm slot. The definition of "slot" by Yuanren Zhao and the definition "frame" and "form" from grammatical study belong to the third level, which is called "paradigm slot". 
In the diagram, the separable slot is formed according to the different ways of the combined or inserted element " $x$ ". "The separable slot" is the basic level of the "slot" system which possesses abstract meaning. Namely, "AB+X" $\rightarrow$ "ABX"—_the combining separable slot; "AB+X" $\rightarrow$ "AXB"—_the inserting separable slot; "AB+X" $\rightarrow$ "AX / BX"—- the elliptical separable slot; "AB+X" $\rightarrow$ "BAX"— the translocated separable slot.

The differences in the formation of $\mathrm{X}$ in each slot and word order determine the pattern which in turn determines the formation of slot patterns. "Separable pattern slot" is the secondary slot which is as abstract as separable slot.

The specific semantic categories of the combined or inserted elements " $x$ " and " $y$ " in each pattern slot determine the unique form of each pattern functions and thereby different separable paradigm slots are formed. Paradigm slot is the third level slot which expresses practical meaning of language. This paper only analyzes some paradigm slots of the combining slots and other slots will be discussed in other paper.

$\mathrm{V}+\mathrm{O}$ separable word $\mathrm{AB}$ and its combined element $\mathrm{X}$ form the combining separable slot " $\mathrm{AB}+\mathrm{X}$ ", namely " $\mathrm{AB}+\mathrm{X}$ " $\rightarrow$ "ABX". According to different forms of " $\mathrm{X}$ " and different word order, four separable pattern slots of separable slots "ABX" are produced, namely "AB" "XAB" "ABx" and "xABy". The separable paradigm slot $\mathrm{ABx}$ is formed of the combining pattern slot $\mathrm{AB}$ with different moods. And according to the different moods of "x", the paradigm slot[ABx] forms six sub-paradigm slots: $[\mathrm{AB}],,[\mathrm{AB} 。],[\mathrm{AB} !],[\mathrm{AB}$ ? ], [ $\mathrm{AB}--]$ and $[\mathrm{AB}$...... $]$. Due to the limited space, this dissertation only take the paradigm slot $[\mathrm{AB}$ ! ] for example. It studies the paradigm slot $[\mathrm{AB}+$ ! ] in a comprehensive perspective, discusses and analyzes the selection and restriction within the quantitative $\mathrm{V}+\mathrm{O}$ separable words. It further analyzes the internal semantic features and rules of use for separable words falling into this category. ${ }^{[3]}$

\section{Analysis of separable paradigm slot $[\mathrm{AB}+$ ! ]}

This paradigm slot leads to different meanings, which is endowed by the formation itself. It shows three meanings: positively imperative mood, exclamation or surprise.

\section{To show imperative mood.}

Selection of separable words which can be used in the paradigm slot [AB! ]

When the paradigm slot shows the positively imperative mood, the explanation of it is that the speaker consciously demands the listener to do something or prompts the listener that something will happen. Therefore, not all of separable words can fall into this paradigm slot. From the perspective of semantics, most of the separable words which can form positively imperative sentences and negatively imperative sentences often show people's action, behaviour, condition, change and so on. They should have the semantic feature of [+autonomous]. However, in the negative imperative sentences, except the autonomous separable words, some individual separable words which have the semantic feature of [-autonomous] also can be used.

From the angle of formation, autonomous and non-autonomous separable words are different in many ways. And the typical difference is "lai" or"qu" can be placed before or after [+autonomous] separable words, but we cannot do the same to [-autonomous] separable words, e.g.
[1]bangmang! (Help!)
[2]※biye ! (※To graduate!)
[1]'laibangmang! (Comehelp!)
$[2]^{\prime} ※$ qubiye ! ( $※$ go to graduate!)
[1]"biebangmang! (Don't help!)
[2]"Don't graduate!
Apply for loan!
※ianzhi! ( $※$ Reduce the value)
go for loan!
biedaikuan! (Don't apply for loan!)
※qubianzhi! (go to reduce the value!)
Stop depreciation!

\footnotetext{
${ }^{33}$ This paper takes $325 \mathrm{~V}+\mathrm{O}$ separable words from the Guideline for Chinese Vocabulary and Character level as the study object (Beijing: Economic \& Science Press, 2001).
} 
Separable words in group 1) have the semantic feature of [+autonomous]. This kind of words can be used in all the imperative sentences no matter they are positive or negative. The words in group[2] have the semantic feature of [-autonomous], and this kind of separable words can be only used in the negatively imperative sencentences. Such distribution of [+autonomous] and [-autonomous] separable words in the imperative sentences mainly has relation with the meaning of their paradigm slot. The meaning of paradigm slot [AB! ]'s positive form is that the speaker consciously demands the listener to do something or prompts the listener to show some certain condition. So the separable words must have the semantic feature of [+autonomous]. And Study shows that the separable words which has semantic feature of [+autonomous] make a great percentage of all the 325 separable words in the research. However, the negative form [bie/bengAB (le )! ] of paradigm slot [AB! ] shows the meaning that the speaker demands the listener not to do something or prompts the listener to avoid the occurrence of some situation. Therefore, generally speaking, the [-autonomous] separable words can be only used in the negatively imperative sentences rather than the positively imperative sentences. ${ }^{[4]}$

It can be seen from the above analysis that nearly all the [+autonomous] separable words can fall into the positively imperative sentences and negatively imperative sentences. However, we found the opposite in the authentic materials. In fact, not all of them can be used in the positively imperative sentences and negatively imperative sentences. Generally speaking, the separable words which have the semantic features of [+autonomous] and [+meliorative] can be used in the positively imperative sentences rather than in the negatively imperative sentences. And most of these separable words indicate mental activities and can be modified by adverbs of degree, e.g.

[3] To make a good show!

[3]'※biezhengqi! (※Don't make effort!)

[3]"Do a very good job.

Work hard!

※bieyonggong! (※Don't work hard!)

very hard working.

From the angle of semantics, single separable words being used in the imperative sentences often have the semantic feature of direction or demand. Because the commendatory terms are rich in emotion and show confirmation or praise, the speaker seldom uses these words to stop or persuade others not to do something. Thus the separable words which have semantic features of [+autonomous] and $[+$ meliorative $]$ are hardly accepted in the negatively imperative sentences. On the contrary, the separable words having the semantic feature of [+autonomous] and [+derogative] cannot be included in the positively imperative sentences in general. They only can be used in negatively imperative sentences. Only the neutral separable words can be freely used, e.g.
[4]※chaozui! (※To quarrel!)
[5]To propose a toast!
[4]'Stop quarreling!
$[5]^{\prime}$ Don't propose a toast!
※ahuo! (※Be angry!)
Don't be angry!
Class begins!
Stop the class!

Separable words in group [4] have the semantic features of [+autonomous] and [+derogative]. From the angle of semantics, since derogative words show negative mood, when the speaker stop the listener to do something or prompt the listener avoid of something, he doesn't show positive evaluation in the negatively imperative sentences. Here "something" often refers to bad behaviour or result. Thus, the separable words having the semantic features of [+autonomous] and [+derogative] are often used in the negatively imperative sentences. Separable words in group [5] have the semantic features of [+autonomous] and [+neutral], and are used freely in the paradigm slots [AB! ]and [别 $\mathrm{AB} !]$

${ }^{[4]}$ Yulin Yuan, Imperative Sentence and the Category of Verb[J], ZhongguoYuwen, 1991, the first volume, P12. 
According to the research, the semantic feature of the separable words which can be used in the paradigm slot [AB! ], namely used in the positively imperative sentences, must be [+autonomous]. However, it doesn't mean that all of separable words having semantic feature of [+autonomous] can be used in this paradigm slot. In other words, it is limited when a separable word form an imperative sentence itself. The words that can be used in the separable slot [AB!] often have the semantic features of [+autonomous] and [-derogative]. But of course there would be exception. According to the research, though the separable words "face to face" and"head on" could be considered as [+autonomous] separable words theoretically, they are almost unacceptable to be used in the paradigm slots $[\mathrm{AB}$ ! ] and [bie/bengAB (le) ! ] in the real context. Only when they become adverbial modifiers and with verbs behind them, they can be used in the imperative sentences, for example: "Talk to him face to face!)| Don't talk to him face to face!| Don't head on!". [-autonomous] separable words "to study together、 to live in the same room、 to work together、not worry、satisfy、sensible、 as one's wish 、 relatively good"etc. are also unacceptable in the imperative sentences.

Analysis of paradigm slot [AB! ]'s explanation

From the angle of semantics, when a separable word form an imperative sentence alone, it mainly shows two meanings: order and prompt.

The imperative sentences which show demand mean that the speaker ask the listener to do something or make the listener stay in some condition. And they can be used at nearly all the occasions in general.e.g.

[6] To be used in the class:

Read the book!

To be used in the military:

Show your opinion!

Sign up the name!

Draw a picture!

Go to war!

Open your mouth!

Fight!

To be used in the administration:

March!

Pay the fines!

Pay reparations!

To be used in daily life:

Go on duty!

Take a shower!

Cut hair!

Go on duty!

Sell goods!

Clap hands!

It can be seen through the research that generally speaking, the separable words having the semantic feature of [+autonomous] often show demand. Comparatively speaking, the separable words are rarely used alone in the military and hard to be popularized. But they are often used in other places and have high probability to be extended.

The imperative sentences showing prompt are often used in such language environment of mentioning others to be careful of something, e.g:

[7] Be careful! (There is ice on the road!)

[8] Be careful! (A car is coming!)

All the above sentences are often used to mention others to pay attention to something in order to prevent unhappy things or harmful things. So this kind of imperative sentences is dependent on the context. These separable words are often mental lexicon, and most of them can be modified by adverbs of degree and can not be overlapped in general, e.g:"very careful ; $※$ liuliushen $\mid ※$ liushenliushen| $※$ zhuzhuyi $\mid ※$ zhuyizhuyi”( $※$ Be careful careful!).

The above study shows that when the separable words are used alone in the imperative sentences, it seems like there is only one word, but in fact, there is a hidden element, that is subject, object or dative, namely "“who'+AB"or" preposition+who'+AB" and so on, e.g:

[9]Broadcasting! (At least 490,000 people had heard her from the broadcast! )

[10]Take a shower! (The kid haven't taken shower for a long time and we must bathe him today! )

[11]Divorce! (I want to divorce because my wife is a bully, and I cannot bear her anymore! )

To show exclamation. When the paradigm slot $[A B !]$ show exclamation, most of separable words can fall into it. However, it is dependent on the context. There must be a following sentence 
which has the emotion of sadness. The meaning of this paradigm slot is dependent on the meaning of the following sentence, e.g.

[12] Suffer losses!(There is nothing she can do, she was really not lucky to meet such a trouble!)

[13] You are lying! (What the teacher said is not interesting at all! )

From the research we can see that both of the two sentences are acceptable. Separable words in group [12] have the semantic meaning of [-autonomous]. Separable words in group [13] have the semantic features of[+autonomous] and [-derogative]. If there are no following sentences, example [12] and[13] are not acceptable. The meaning of this paradigm slot relys on the following sentence.

To show surprise. When the paradigm slot $[A B$ ! ] shows surprise, most of separable words can be used in it. However, it is dependent on the context. There must be a following sentence which has the emotion of disbelif or unpredictable. The meaning of this paradigm slot is dependent on the following sentence, e.g.

[14] He missed the class?(It is impossible. He is really a good student.)

[15]He got hurt? (He was fine a few minutes ago.)

From the research we can see that both of the two sentences are acceptable. Separable words in group [14] have the semantic features of [+autonomous] and [-derogative]. Separable words in group [15] have the semantic feature of [-autonomous]. If there are no following sentences, example [14] and [15] are not acceptable. The meaning of this paradigm slot relys on the following sentence.

\section{Conclusion}

Paradigm slot [AB ! ] leads to different meanings, and it shows imperative mood, exclamation and surprise.

\section{When the paradigm slot [AB ! ] shows imperative mood:.}

- it can only express the positive meaning;

- separable words which can be used in it often have the semantic featurs of [+autonomous] and [-derogative];

- Most of the separable words used here show mental activities;

- Most of separable words having the semantic feature of [+autonomous] can show demand. But they are seldom used alone in the imperative sentence about military, they are often used in other occurrence;

- When a separable word forms the imperative itself, it seems that there is only one word, however, there is an elliptical element which could be subject, object or dative, namely "“who'+AB" or " " preposition+who'+AB";

- When this paradigm show imperative of prompting, the separable words often show mental activities. They often can be modified by adverbs of degree and cannot be overlapped.

When the paradigm slot [AB ! ] shows exclamation or surprise:.

Nearly all the separable words can be used in this paradigm slot, but at that time, the paradigm slot show a great dependence on the context.

\section{Acknowledgment}

This study has gotten the support of the national social science fund major projects (the grant number is $11 \&$ ZD189).

\section{References}

[1] Yuanren Zhao, A Grammar of Spoken Chinese,Commercial Press, Beijing,1979, pp.7.

[2] Yulin Yuan, Imperative Sentence and the Category of Verb, ZhongguoYuwen, 1991, the first volume, pp.12. 
[3] Chunling Li,A Study on the "V+O" Separable Words \& Its Separable Slots in Modern Chinese, Doctoral dissertation of Wuhan University, 2008, pp.20-35.

[4] Qingzhu Ma, Controllable and Uncontrollable Verbs, Journal of Chinese Linguistics Monograph Series, march 1988.

[5] Z.W.Feng, "Machine Translation," China Translation and Publishing Corporation, 1998, pp.412-434. 